\title{
Intermediate Osteogenic Neoplasm
}

National Cancer Institute

\section{Source}

National Cancer Institute. Intermediate Osteogenic Neoplasm. NCI Thesaurus. Code C121925.

A non-metastasizing, locally aggressive, bone-forming neoplasm. 